\title{
Effects of nuclear symmetry energy and equation of state on neutron star properties
}

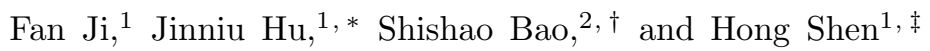 \\ ${ }^{1}$ School of Physics, Nankai University, Tianjin 300071, China \\ ${ }^{2}$ School of Physics and Information Engineering, \\ Shanxi Normal University, Linfen 041004, China
}

\begin{abstract}
We study the effects of nuclear symmetry energy on the mass-radius relation and tidal deformability of neutron stars, considering the self-consistency of the equation of state (EOS). We first construct a set of unified EOSs based on relativistic mean-field models with different density dependence of the symmetry energy. For the description of pasta phases appearing in the inner crust of neutron stars, we perform a self-consistent Thomas-Fermi calculation using the same nuclear interaction as that for the uniform matter in the core. To examine possible effects from the self-consistency of the EOS on neutron-star properties, we separately investigate the impacts of crust and core segments. By matching the same core EOS to different crust EOSs, it is found that neutron-star radii are significantly affected by the crust segment. On the other hand, the neutron-star radii are also strongly dependent on the core EOS. However, the correlation between the radius and the symmetry energy slope of the core EOS is opposite to that of the crust EOS. It is likely that the nuclear model with a small slope parameter is favored by recent astrophysical observations.
\end{abstract}

PACS numbers: 26.60.-c, 26.60.Gj, 21.65.Cd

Keywords: Symmetry energy, Pasta phase, Neutron stars

\section{INTRODUCTION}

The recent detection of gravitational waves from a binary neutron-star merger, known as GW170817, provides an upper limit on the tidal deformability of neutron stars [1, 2], which can be used to constrain the equation of state (EOS) of dense matter. Another strong constraint on the EOS comes from the observations of massive pulsars, PSR J1614-2230 [3, 4], PSR J0348+0432 [5], and PSR J0740+6620 [6], which requires the maximum neutron-star mass to be larger than $\approx 2 M_{\odot}$. The EOS plays a decisive role in understanding various properties of neutron stars [7-10]. Generally, the EOS used for the calculations of neutron-star structure must cover a wide density range that can be divided into three segments: (a) the EOS of the outer crust below the neutron drip density; (b) the EOS of the inner crust from neutron drip to crust-core transition; (c) the EOS of the liquid core above the crust-core transition. The outer crust, which extends from the surface of the star to the neutron drip density, is composed of spherical nuclei arranged in a lattice and a background of relativistic electron gas. The behavior of the outer crust is mainly determined by experimental nuclear masses, and therefore no significant differences exist in the EOS of the outer crust when using different nuclear-mass models [11]. With increasing depth in the neutron star, neutrons drip out of nuclei and form a dilute neutron gas together with the electron gas in the inner crust. As the density increases

\footnotetext{
*Electronic address: hujinniu@nankai.edu.cn

$\dagger$ Electronic address: bao shishao@163.com

${ }^{\ddagger}$ Electronic address: shennankai@gmail.com
}

toward the crust-core transition, spherical nuclei may become unstable and nuclear shape is likely to change from droplet to rod, slab, tube, and bubble, known as nuclear pasta phases [12 14]. The inner crust of neutron stars has received much attention due to its complex structure and important role in astrophysical observations [15 18]. The transition from the inner crust to the core occurs at about $1 / 3$ to $1 / 2$ nuclear saturation density depending on the nuclear interaction used. The uniform matter in the core consists of a mixture of neutrons, protons, electrons, and muons in $\beta$ equilibrium, which extends from the crust-core transition to the center of the star. In the deep interior of neutron stars, non-nucleonic degrees of freedom, such as hyperons and quarks, may appear to soften the EOS, as extensively discussed in the literature [10, 19 22]. In the inner core region of massive neutron stars, the baryon density can reach higher than five times nuclear saturation density, where the deconfinement hadron-quark phase transition probably occurs. The phase transition from hadronic matter to quark matter is often assumed to proceed with the Gibbs or Maxwell constructions, depending on the surface tension at the hadron-quark interface [23]. In addition, the hadron-quark pasta phases could be formed as a result of the competition between the Coulomb and surface energies, as discussed in Refs. [20, 24 26]. The appearance of a hadron-quark phase transition would lead to a reduction of the maximum neutron-star mass, but it is still possible to be compatible with the $\approx 2 M_{\odot}$ constraint 24 26]. For simplicity, we do not include non-nucleonic degrees of freedom in the present study.

It is important to investigate neutron-star properties using the unified EOS, where both the core and the crust are based on the same nuclear interaction model. 
There are several works 27 32] on developing the unified EOS. A compressible liquid drop model was used to describe the nuclei in the crust in Ref. 27], whereas the parameterized Thomas-Fermi method [28, 29] and selfconsistent Thomas-Fermi approximation 30-32] were employed for nonuniform matter in the crust region. The use of a unified EOS is important for the description of the crust-core transition and detailed properties of neutron stars. However, in most calculations, a nonunified EOS is employed, i.e., the core EOS is matched to a crust EOS obtained from different models. It was found in Ref. [32] that the matching procedure could slightly affect the resulting radius and crust thickness of neutron stars. It is often argued that the crust EOS has less effect on the global properties of neutron stars. Considering recent observational progress related to neutron stars, we would like to quantitatively examine the effect of matching different crust EOSs to the core EOS on neutron-star properties such as the radius and tidal deformability. For this purpose, we construct a set of EOSs for both the inner crust and the core, employing the relativistic meanfield (RMF) model [33 35].

It is well known that the nuclear symmetry energy and its density dependence play an important role in understanding many phenomena in nuclear physics and astrophysics [9, 10, 36 38]. It has been found that various properties of neutron stars, such as the radius and the crust structure, are sensitive to the symmetry energy $E_{\text {sym }}$ and its slope parameter $L[9,15,17,39-41]$. In recent decades, great efforts have been devoted to constraining the values of $E_{\mathrm{sym}}$ and $L$ at saturation density based on astrophysical observations and terrestrial nuclear experiments [42 49]. In Ref. 10], a sufficient number of constraints on the symmetry energy parameters have been summarized, and the most probable values for the symmetry energy and its slope at saturation density were found to be $E_{\text {sym }}=31.7 \pm 3.2 \mathrm{MeV}$ and $L=58.7 \pm 28.1 \mathrm{MeV}$, respectively, with a much larger error for $L$ than that for $E_{\mathrm{sym}}$. To study the effect of the symmetry energy on neutron-star properties, we employ a set of generated RMF models based on the TM1 parametrization, which was described in our previous work [50]. The original TM1 model [35] could provide satisfactory description for finite nuclei, and meanwhile it has also been successfully used to construct the EOS for supernova simulations and neutron stars [10, 28, 51]. We introduce an additional $\omega-\rho$ coupling term in the TM1 model, which plays an essential role in controlling the density dependence of the symmetry energy [40, 41, 50, 52]. By adjusting simultaneously two parameters associated to the $\rho$ meson $\left(g_{\rho}\right.$ and $\left.\Lambda_{\mathrm{v}}\right)$, we can generate a model with a given $L$ at saturation density and a fixed $E_{\mathrm{sym}}$ at a density of $0.11 \mathrm{fm}^{-3}$. The choice of fixing symmetry energy at $0.11 \mathrm{fm}^{-3}$ is based on the following consideration. The generated models with different $L$ should be able to provide results for finite nuclei similar to the original TM1 model. It is well known that the binding energy of finite nuclei is essentially de- termined by the symmetry energy at $\approx 0.11 \mathrm{fm}^{-3}$, not by the symmetry energy at saturation density. Therefore, the value of the symmetry energy at $\approx 0.11 \mathrm{fm}^{-3}$ is well constrained by experimental nuclear masses. By keeping $E_{\text {sym }}$ fixed at $n_{b}=0.11 \mathrm{fm}^{-3}$, the resulting binding energies of finite nuclei are almost unchanged within the set of generated models. This is because the average value of the density in finite nuclei is about $0.11 \mathrm{fm}^{-3}$. It is noteworthy that all models in this set have the same isoscalar properties and fixed symmetry energy at $n_{b}=0.11 \mathrm{fm}^{-3}$, but they have different density dependence of the symmetry energy. Therefore, this set of models is suitable for studying the correlations between the slope parameter $L$ and neutron-star properties. In the present work, we use RMF models based on the TM1 parametrization for the calculations of neutron-star properties, which may introduce some model dependency in the results. Generally, the predicted properties of neutron stars, such as gravitational masses and radii, are model dependent, as can be found in Refs. 9, 10, 32, 53]. As an example, the radius of a canonical $1.4 M_{\odot}$ neutron star $\left(R_{1.4}\right)$ varies between $\approx 11$ and $15 \mathrm{~km}$ for some popular EOSs supporting the $\approx 2 M_{\odot}$ maximum mass constraint [9, 10, 32, 53]. Even for several models with a similar slope parameter $L$, the difference in $R_{1.4}$ can be as large as $\approx 1 \mathrm{~km}$. Therefore, the slope parameter $L$ cannot be precisely constrained by observations of neutron-star radii. However, a positive correlation between the slope parameter $L$ and neutronstar radius is consistent among different models, which will be studied using the RMF models in the present calculations.

We have two aims in this study. The first is to construct a set of unified EOSs using the RMF models that have the same isoscalar properties but different density dependence of the symmetry energy, and then apply these EOSs to study the effects of the symmetry energy on neutron-star properties. The second is to examine separately the influences from the crust and core segments on the radius and tidal deformability of neutron stars. By matching different crust EOSs to a fixed core EOS, the uncertainty induced by the crust segment in a nonunified EOS can be estimated quantitatively. For constructing a unified EOS, we perform the self-consistent ThomasFermi calculations for pasta phases appearing in the inner crust, and then judge the crust-core transition by comparing the energy densities between pasta phases and homogeneous matter. Since the same nuclear model is employed for the description of the two phases, the crust-core transition is determined in a consistent manner and the resulting unified EOS is quite smooth. In the present work, the Thomas-Fermi approximation is used only for the inner crust but not for the outer crust. This is because the shell effect is not considered within the Thomas-Fermi approximation. In fact, when the BaymPethick-Sutherland (BPS) EOS [54] for the outer crust is replaced by the one obtained from the Thomas-Fermi calculation, no significant difference is observed in the star radius. Therefore, we prefer to use the BPS EOS 
for the outer crust below the neutron drip density in the present calculations.

The recent GW170817 event triggered extensive studies for constraining the EOS from measurements of the tidal deformability in a binary neutron-star system $55-$ 65]. The analysis of GW170817 data provides valuable constraints on the tidal deformabilities of the binary neutron-star merger [1, 2]. The correlation between the symmetry energy and the tidal deformability was recently investigated within various frameworks 62 65]. In the present work, we use a set of unified EOSs to compute the tidal deformability of neutron stars and study its dependence on the symmetry energy slope $L$. Furthermore, we match different crust and core segments in order to examine their influence on the resulting tidal deformability.

This article is organized as follows. In Sec. II we briefly describe the RMF model and the self-consistent Thomas-Fermi approximation used for constructing the EOS. In Sec. III we show the effects of the symmetry energy on neutron-star properties using the unified EOS. Furthermore, the influences from the crust and core segments are examined separately using two sets of nonunified EOSs. Section IV is devoted to the conclusions.

\section{FORMALISM}

We construct the EOS of neutron-star matter employing the RMF model for nuclear interactions. In the RMF approach [33 35], nucleons interact through the exchange of various mesons, including the isoscalar-scalar meson $\sigma$, the isoscalar-vector meson $\omega$, and the isovector-vector meson $\rho$. For a system consisting of neutrons, protons, electrons, and muons, the Lagrangian density reads

$$
\begin{aligned}
\mathcal{L}_{\mathrm{RMF}}= & \sum_{b=p, n} \bar{\psi}_{b}\left\{i \gamma_{\mu} \partial^{\mu}-\left(M+g_{\sigma} \sigma\right)\right. \\
& \left.-\gamma_{\mu}\left[g_{\omega} \omega^{\mu}+\frac{g_{\rho}}{2} \tau_{a} \rho^{a \mu}+\frac{e}{2}\left(1+\tau_{3}\right) A^{\mu}\right]\right\} \psi_{b} \\
& +\frac{1}{2} \partial_{\mu} \sigma \partial^{\mu} \sigma-\frac{1}{2} m_{\sigma}^{2} \sigma^{2}-\frac{1}{3} g_{2} \sigma^{3}-\frac{1}{4} g_{3} \sigma^{4} \\
& -\frac{1}{4} W_{\mu \nu} W^{\mu \nu}+\frac{1}{2} m_{\omega}^{2} \omega_{\mu} \omega^{\mu}+\frac{1}{4} c_{3}\left(\omega_{\mu} \omega^{\mu}\right)^{2} \\
& -\frac{1}{4} R_{\mu \nu}^{a} R^{a \mu \nu}+\frac{1}{2} m_{\rho}^{2} \rho_{\mu}^{a} \rho^{a \mu}-\frac{1}{4} F_{\mu \nu} F^{\mu \nu} \\
& +\Lambda_{\mathrm{v}}\left(g_{\omega}^{2} \omega_{\mu} \omega^{\mu}\right)\left(g_{\rho}^{2} \rho_{\mu}^{a} \rho^{a \mu}\right) \\
& +\sum_{l=e, \mu} \bar{\psi}_{l}\left(i \gamma_{\mu} \partial^{\mu}-m_{l}+e \gamma_{\mu} A^{\mu}\right) \psi_{l},
\end{aligned}
$$

where $W^{\mu \nu}, R^{a \mu \nu}$, and $F^{\mu \nu}$ are the antisymmetric field tensors corresponding to $\omega^{\mu}, \rho^{a \mu}$, and $A^{\mu}$, respectively. In a static system within the mean-field approximation, the nonvanishing meson mean fields are $\sigma=\langle\sigma\rangle, \omega=$ $\left\langle\omega^{0}\right\rangle, \rho=\left\langle\rho^{30}\right\rangle$, and $A=\left\langle A^{0}\right\rangle$. The chemical potentials of nucleons are given by

$$
\begin{aligned}
& \mu_{p}=\sqrt{\left(k_{F}^{p}\right)^{2}+M^{* 2}}+g_{\omega} \omega+\frac{g_{\rho}}{2} \rho+e A, \\
& \mu_{n}=\sqrt{\left(k_{F}^{n}\right)^{2}+M^{* 2}}+g_{\omega} \omega-\frac{g_{\rho}}{2} \rho,
\end{aligned}
$$

where $M^{*}=M+g_{\sigma} \sigma$ is the effective nucleon mass and $k_{F}^{i}$ is the Fermi momentum of species $i$, which is related to the number density by $n_{i}=\left(k_{F}^{i}\right)^{3} / 3 \pi^{2}$. It is noteworthy that the $\omega-\rho$ coupling term plays an important role in determining the density dependence of the symmetry energy [40, 41, 50, 52]. The symmetry energy of nuclear matter is expressed as

$$
\begin{aligned}
E_{\mathrm{sym}} & =\frac{1}{2}\left[\frac{\partial^{2}\left(\varepsilon / n_{b}\right)}{\partial \alpha^{2}}\right]_{\alpha=0} \\
& =\frac{k_{F}^{2}}{6 \sqrt{k_{F}^{2}+M^{* 2}}}+\frac{g_{\rho}^{2} n_{b}}{8\left(m_{\rho}^{2}+2 \Lambda_{\mathrm{v}} g_{\rho}^{2} g_{\omega}^{2} \omega^{2}\right)},
\end{aligned}
$$

with $\alpha=\left(n_{n}-n_{p}\right) / n_{b}$ being the asymmetry parameter. The slope of the symmetry energy is given by

$$
L=3 n_{0}\left[\frac{\partial E_{\mathrm{sym}}\left(n_{b}\right)}{\partial n_{b}}\right]_{n_{b}=n_{0}} .
$$

We use a set of generated models based on the TM1 parametrization [50], where the coupling constants, $g_{\rho}$ and $\Lambda_{\mathrm{v}}$, are simultaneously adjusted so as to achieve a given symmetry energy slope $L$ at saturation density $n_{0}$ while keeping the symmetry energy $E_{\text {sym }}$ fixed at a density of $0.11 \mathrm{fm}^{-3}$. It was shown in Ref. [50] that all models in the set could provide the same isoscalar properties and similar binding energies of finite nuclei as the original TM1 model, but have different symmetry energy slope $L$. To make the paper self-contained, we list in Table \ the model parameters and saturation properties, while the calculated properties of ${ }^{208} \mathrm{~Pb}$ are shown in the last three lines. It is found that the models with different $L$ predict very similar binding energy per nucleon and charge radius for ${ }^{208} \mathrm{~Pb}$, whereas the neutron-skin thickness $\triangle r_{\mathrm{np}}$ $\left({ }^{208} \mathrm{~Pb}\right)$ obviously increases with increasing $L$. We show in Fig. 1 the symmetry energy $E_{\text {sym }}$ as a function of the baryon density $n_{b}$ for all models listed in Table I. It is seen that the set of models has the same $E_{\mathrm{sym}}$ at a density of $0.11 \mathrm{fm}^{-3}$, but different values of $E_{\mathrm{sym}}$ at lower and higher densities due to different slope $L$. The behavior of $E_{\text {sym }}$ plays a crucial role in determining several properties of neutron stars.

The npe $\mu$ matter in the neutron-star core has a uniform density distribution under the conditions of $\beta$ equilibrium and charge neutrality. The dense core EOS can be achieved by solving a set of coupled equations in the RMF model. As the density decreases to about $n_{0} / 2$, the crust-core transition occurs, where nucleons cluster into pasta phases or spherical nuclei. This is because the uniform matter is energetically unstable against cluster formation at low densities. For nonuniform matter in the 
TABLE I: Parameter sets used in this work and corresponding saturation properties. The quantities $E_{0}, K, E_{\text {sym }}$, and $L$ are, respectively, the energy per nucleon, incompressibility coefficient, symmetry energy, and symmetry energy slope at saturation density $n_{0}$. The last three lines show the neutron-skin thickness $\Delta r_{\mathrm{np}}$, charge radius $r_{\mathrm{c}}$, and binding energy per nucleon $E / A$ of ${ }^{208} \mathrm{~Pb}$. The models are generated from the original TM1 model $(L=111)$ by tuning $g_{\rho}$ and $\Lambda_{\mathrm{v}}$ to achieve a given slope $L$ at $n_{0}$ and a fixed symmetry energy $E_{\text {sym }}=28.05 \mathrm{MeV}$ at a density of $0.11 \mathrm{fm}^{-3}$. Differences among these models are shown in bold. Nucleon and meson masses are given in Refs. [35, 50].

\begin{tabular}{lcccc}
\hline \hline Model & TM1 $(L=40)$ & TM1 $(L=60)$ & TM1 $(L=80)$ & TM1 $(L=111)$ \\
\hline$g_{\sigma}$ & 10.0289 & 10.0289 & 10.0289 & 10.0289 \\
$g_{\omega}$ & 12.6139 & 12.6139 & 12.6139 & 12.6139 \\
$g_{2}\left(\mathrm{fm}^{-1}\right)$ & -7.2325 & -7.2325 & -7.2325 & -7.2325 \\
$g_{3}$ & 0.6183 & 0.6183 & 0.6183 & 0.6183 \\
$c_{3}$ & 71.3075 & 71.3075 & 71.3075 & 71.3075 \\
$g_{\rho}$ & $\mathbf{1 3 . 9 7 1 4}$ & $\mathbf{1 1 . 2 6 1 0}$ & $\mathbf{1 0 . 1 4 8 4}$ & $\mathbf{9 . 2 6 4 4}$ \\
$\Lambda_{\mathrm{v}}$ & $\mathbf{0 . 0 4 2 9}$ & $\mathbf{0 . 0 2 4 8}$ & $\mathbf{0 . 0 1 2 8}$ & $\mathbf{0 . 0 0 0 0}$ \\
\hline$n_{0}\left(\mathrm{fm}{ }^{-3}\right)$ & 0.145 & 0.145 & 0.145 & 0.145 \\
$E_{0}(\mathrm{MeV})$ & -16.3 & -16.3 & -16.3 & -16.3 \\
$K(\mathrm{MeV})$ & 281 & 281 & 281 & 281 \\
$E_{\mathrm{sym}}(\mathrm{MeV})$ & $\mathbf{3 1 . 3 8}$ & $\mathbf{3 3 . 2 9}$ & $\mathbf{3 4 . 8 6}$ & $\mathbf{3 6 . 8 9}$ \\
$L(\mathrm{MeV})$ & $\mathbf{4 0}$ & $\mathbf{6 0}$ & $\mathbf{8 0}$ & $\mathbf{1 1 1}$ \\
\hline$\triangle r_{\mathrm{np}}\left({ }^{208} \mathrm{~Pb}\right)(\mathrm{fm})$ & $\mathbf{0 . 1 6}$ & $\mathbf{0 . 2 1}$ & $\mathbf{0 . 2 4}$ & $\mathbf{0 . 2 7}$ \\
$r_{\mathrm{c}}\left({ }^{208} \mathrm{~Pb}\right)(\mathrm{fm})$ & $\mathbf{5 . 5 6}$ & $\mathbf{5 . 5 5}$ & $\mathbf{5 . 5 4}$ & $\mathbf{5 . 5 4}$ \\
$E / A\left({ }^{208} \mathrm{~Pb}\right)(\mathrm{MeV})$ & 7.88 & 7.88 & 7.88 & 7.88 \\
\hline \hline
\end{tabular}

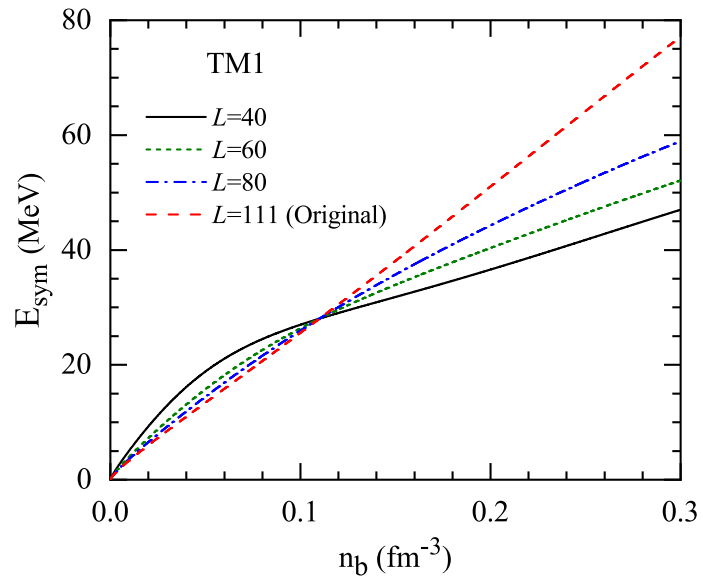

FIG. 1: (Color online) Symmetry energy $E_{\text {sym }}$ as a function of the baryon density $n_{b}$ for the generated TM1 models with different slope parameter $L$. The symmetry energy is fixed at a density of $0.11 \mathrm{fm}^{-3}$.

inner crust, we perform a self-consistent Thomas-Fermi calculation as described in our previous work [17]. The Wigner-Seitz cell approximation is adopted to simplify the calculation of pasta phases. The stable cell shape, which is determined by minimizing the energy density at a given density $n_{b}$, may change from droplet to rod, slab, tube, and bubble as the density increases. For simplicity, we assume the electron density is uniform throughout the Wigner-Seitz cell. In the Thomas-Fermi approximation, the total energy per cell is calculated from

$$
E_{\text {cell }}=\int_{\text {cell }} \varepsilon_{\text {rmf }}(\mathbf{r}) d \mathbf{r}+\varepsilon_{e} V_{\text {cell }},
$$

where $\varepsilon_{\text {rmf }}(\mathbf{r})$ is the local energy density at position $\mathbf{r}$ given in the RMF model and $\varepsilon_{e}$ is the kinetic energy density of electrons. We consider different pasta configurations including the droplet, rod, slab, tube, and bubble. The volume of the Wigner-Seitz cell for different configurations is expressed as

$$
V_{\text {cell }}= \begin{cases}\frac{4}{3} \pi r_{\mathrm{ws}}^{3} & (\text { droplet and bubble }) \\ l \pi r_{\mathrm{ws}}^{2} & (\text { rod and tube }) \\ 2 r_{\mathrm{ws}} l^{2} & (\text { slab })\end{cases}
$$

where $r_{\mathrm{ws}}$ is the radius of a spherical cell for the droplet and bubble configurations, while the rod and tube have cylindrical shapes with radius $r_{\text {ws }}$ and length $l$ and the slab has width $l$ and thickness $2 r_{\text {ws }}$. Actually, the energy density of the system would not be affected by the choices of the length for a cylindrical shape and the width for a slab. At a given average baryon density $n_{b}$, we minimize the total energy density $\varepsilon=E_{\text {cell }} / V_{\text {cell }}$ with respect to the cell size $r_{\mathrm{ws}}$ for each pasta configuration, and then determine the energetically favored state with the lowest energy density. The pressure is calculated from the 
thermodynamic relation

$$
P=\sum_{i=b, l} \mu_{i} n_{i}-\varepsilon .
$$

The crust-core transition occurs at the density where the energy density of the homogeneous phase becomes lower than that of the pasta phase. It is well known that the symmetry energy slope $L$ plays an important role in determining the pasta phase structure and the crust-core transition [15, 17, 39]. In Table II we present the onset densities of various nonspherical nuclei and homogeneous matter for the generated TM1 models with different $L$. It is seen that as $L$ increases, the crust-core transition density (i.e., the onset density of homogeneous matter) significantly decreases and some pasta phases disappear. The model with $L=40 \mathrm{MeV}$ predicts the transition from droplet to rod occurs at $n_{b} \approx 0.049 \mathrm{fm}^{-3}$, then the pasta phases of slab, tube, and bubble appear one by one, and finally transition to homogeneous matter occurs at $n_{b} \approx 0.099 \mathrm{fm}^{-3}$. For the original TM1 model with $L=111 \mathrm{MeV}$, only the droplet configuration appears in the inner crust, and the transition from droplet to homogeneous matter occurs at $n_{b} \approx 0.062 \mathrm{fm}^{-3}$. We note that the inner crust are calculated in the Thomas-Fermi approximation for the density region between the neutron drip and the crust-core transition. For the outer crust, we use the well-known BPS EOS, which is matched to the inner-crust EOS at the neutron drip density.

TABLE II: Onset densities given in the unit of $\mathrm{fm}^{-3}$ for various nonspherical nuclei (rod, slab, tube, and bubble) and homogeneous matter (HM) obtained in the generated TM1 models with different $L$.

\begin{tabular}{lccccc}
\hline \hline Model & Rod & Slab & Tube & Bubble & HM \\
\hline $\operatorname{TM} 1(L=40)$ & 0.049 & 0.064 & 0.082 & 0.089 & 0.099 \\
$\operatorname{TM} 1(L=60)$ & 0.066 & 0.076 & 0.081 & & 0.083 \\
$\operatorname{TM} 1(L=80)$ & & & & & 0.072 \\
$\operatorname{TM} 1(L=111)$ & & & & & 0.062 \\
\hline \hline
\end{tabular}

We apply the EOS constructed above to calculate the mass and radius of a neutron star by solving the TolmanOppenheimer-Volkoff (TOV) equation in units of $G=$ $c=1$,

$$
\begin{aligned}
\frac{d P(r)}{d r}= & -\frac{M(r) \varepsilon(r)}{r^{2}}\left[1+\frac{P(r)}{\varepsilon(r)}\right] \\
& \times\left[1+\frac{4 \pi r^{3} P(r)}{M(r)}\right]\left[1-\frac{2 M(r)}{r}\right]^{-1} \\
\frac{d M(r)}{d r}= & 4 \pi r^{2} \varepsilon(r)
\end{aligned}
$$

where $P(r)$ and $\varepsilon(r)$ are the pressure and energy density at the radial coordinate $r$, respectively. $M(r)$ is the gravitational mass enclosed within the radius $r$. The dimensionless tidal deformability of a neutron star is expressed as 66,67$]$

$$
\Lambda=\frac{2}{3} k_{2} C^{-5},
$$

where $C=M / R$ is the compactness parameter of the star with mass $M$ and radius $R$. The tidal Love number $k_{2}$ is calculated from

$$
\begin{aligned}
k_{2}= & \frac{8 C^{5}}{5}(1-2 C)^{2}\left[2-y_{R}+2 C\left(y_{R}-1\right)\right] \\
& \times\left\{2 C\left[6-3 y_{R}+3 C\left(5 y_{R}-8\right)\right]\right. \\
& +4 C^{3}\left[13-11 y_{R}+C\left(3 y_{R}-2\right)+2 C^{2}\left(1+y_{R}\right)\right] \\
& +3(1-2 C)^{2}\left[2-y_{R}+2 C\left(y_{R}-1\right)\right] \\
& \times \ln (1-2 C)\}^{-1}
\end{aligned}
$$

where $y_{R}=y(R)$ is obtained by solving the following differential equation:

$$
r \frac{d y(r)}{d r}+y(r)^{2}+y(r) F(r)+r^{2} Q(r)=0
$$

with

$$
\begin{aligned}
F(r)= & \left\{1-4 \pi r^{2}[\varepsilon(r)-P(r)]\right\}\left[1-\frac{2 M(r)}{r}\right]^{-1}, \quad(14) \\
Q(r)= & 4 \pi\left[5 \varepsilon(r)+9 P(r)+\frac{\varepsilon(r)+P(r)}{\partial P(r) / \partial \varepsilon(r)}-\frac{6}{4 \pi r^{2}}\right] \\
& \times\left[1-\frac{2 M(r)}{r}\right]^{-1}-\frac{4 M(r)^{2}}{r^{4}}\left[1+\frac{4 \pi r^{3} P(r)}{M(r)}\right]^{2} \\
& \times\left[1-\frac{2 M(r)}{r}\right]^{-2} .
\end{aligned}
$$

In a binary neutron-star system, the tidal effect is given by the combined dimensionless tidal deformability

$$
\tilde{\Lambda}=\frac{16}{13} \frac{(12 q+1) \Lambda_{1}+(12+q) q^{4} \Lambda_{2}}{(1+q)^{5}},
$$

where $\Lambda_{1}$ and $\Lambda_{2}$ are the individual tidal deformabilities of the two neutron stars with the mass ratio $q=$ $M_{2} / M_{1} \leq 1$

\section{RESULTS AND DISCUSSION}

We present numerical results for neutron-star properties using the EOSs obtained with the set of RMF models. To examine the effects of the symmetry energy, we apply the unified EOS to compute various properties of neutron stars. In order to separately investigate the influence of crust and core segments, nonunified EOSs are used by matching different crust and core EOSs.

\section{A. Neutron-star properties with unified EOSs}

The unified EOS used in this work is obtained by performing a self-consistent Thomas-Fermi calculation for 


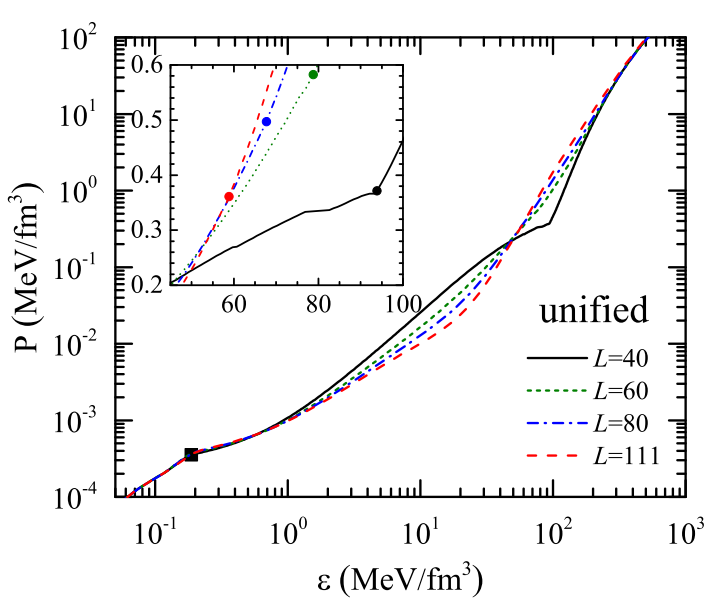

FIG. 2: (Color online) Pressure $P$ as a function of the energy density $\varepsilon$ obtained using the set of generated TM1 models with different $L$ for the inner crust and core. The BPS EOS is adopted for the outer crust and the matching point is marked by the filled square. The crust-core transition is indicated by the filled circles in the inset.

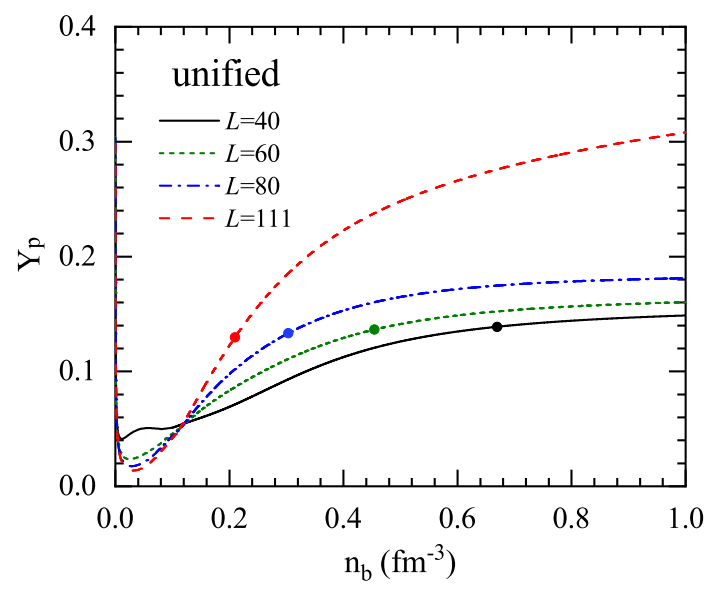

FIG. 3: (Color online) Proton fraction $Y_{p}$ of the unified EOSs as a function of the baryon density $n_{b}$ for the set of generated TM1 models. The filled circles indicate the threshold for the dUrca process.

the inner crust, which is smoothly connected to the core EOS based on the same nuclear model. We use the BPS EOS for the outer crust below the neutron drip density. In Fig. 2, we plot the pressure $P$ as a function of the energy density $\varepsilon$ obtained using the set of generated TM1 models with different slope parameters $L$. The crustcore transition is indicated by the filled circles. It is shown that the model with a small value of $L$ predicts a large crust-core transition and relatively small pressures at high densities. In the Thomas-Fermi approximation,

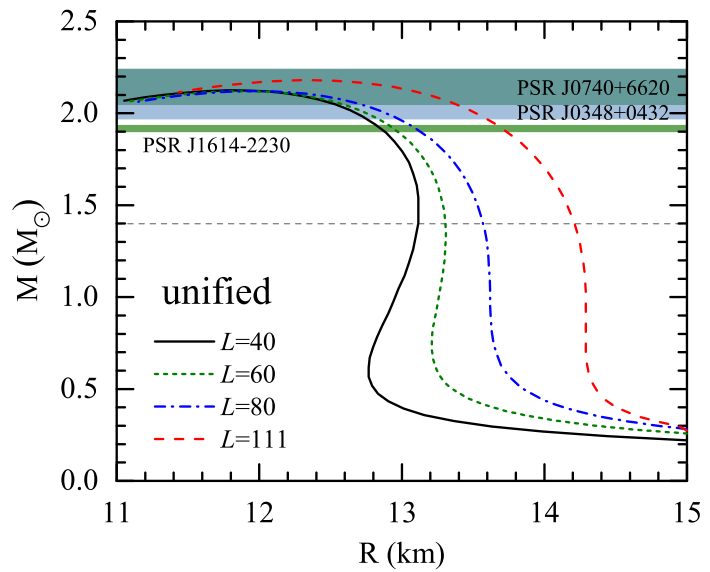

FIG. 4: (Color online) Mass-radius relations of neutron stars obtained using the unified EOSs shown in Fig. 2. The horizontal bars indicate the recent neutron-star mass measurements of PSR J1614-2230 [3, 4], PSR J0348+0432 [5], and PSR J0740+6620 []] .

the phase transition is determined by minimizing the energy density. As a result, the energy density is a smooth function of the baryon density, but the pressure as the first derivative of the energy may exhibit a weak discontinuity of first-order phase transition 68]. In Fig. 2] a clear kink in the $\operatorname{TM} 1(L=40)$ EOS is observed at the crust-core transition, whereas it is invisible in other cases. This is because the $\operatorname{TM} 1(L=40)$ EOS has relatively small pressure and large crust-core transition density.

It is well known that the most efficient mechanism for neutron-star cooling is the direct Urca (dUrca) process, i.e., the electron capture by a proton and the beta decay of a neutron. The threshold for the dUrca process is mainly determined by the proton fraction $Y_{p}$ in the cores of neutron stars, where the proton fraction is large enough to allow for momentum conservation. In simple npe neutron-star matter, the dUrca process can occur for $Y_{p} \geq 1 / 9$. When muons are included under the equilibrium condition $\mu_{e}=\mu_{\mu}$, the critical $Y_{p}$ for the dUrca process is in the range of $(11.1-14.8) \%$ 69]. In fact, the proton fraction $Y_{p}$ of neutron-star matter is strongly dependent on the symmetry energy. In Fig. 3, the proton fraction $Y_{p}$ of the unified EOSs is plotted as a function of the baryon density $n_{b}$ for the set of generated TM1 models, and the corresponding threshold for the dUrca process is indicated by the filled circles. These models show different behaviors of the symmetry energy due to different slope parameters $L$. The model with $L=40$ $\mathrm{MeV}$ predicts a small $Y_{p}$ at high densities and a large threshold density of $0.67 \mathrm{fm}^{-3}$ for the dUrca process. In contrast, the original TM1 model $(L=111 \mathrm{MeV})$ gives a much higher $Y_{p}$ and small threshold density of $0.21 \mathrm{fm}^{-3}$. It has been reported in Ref. [70] that neutron-star cool- 

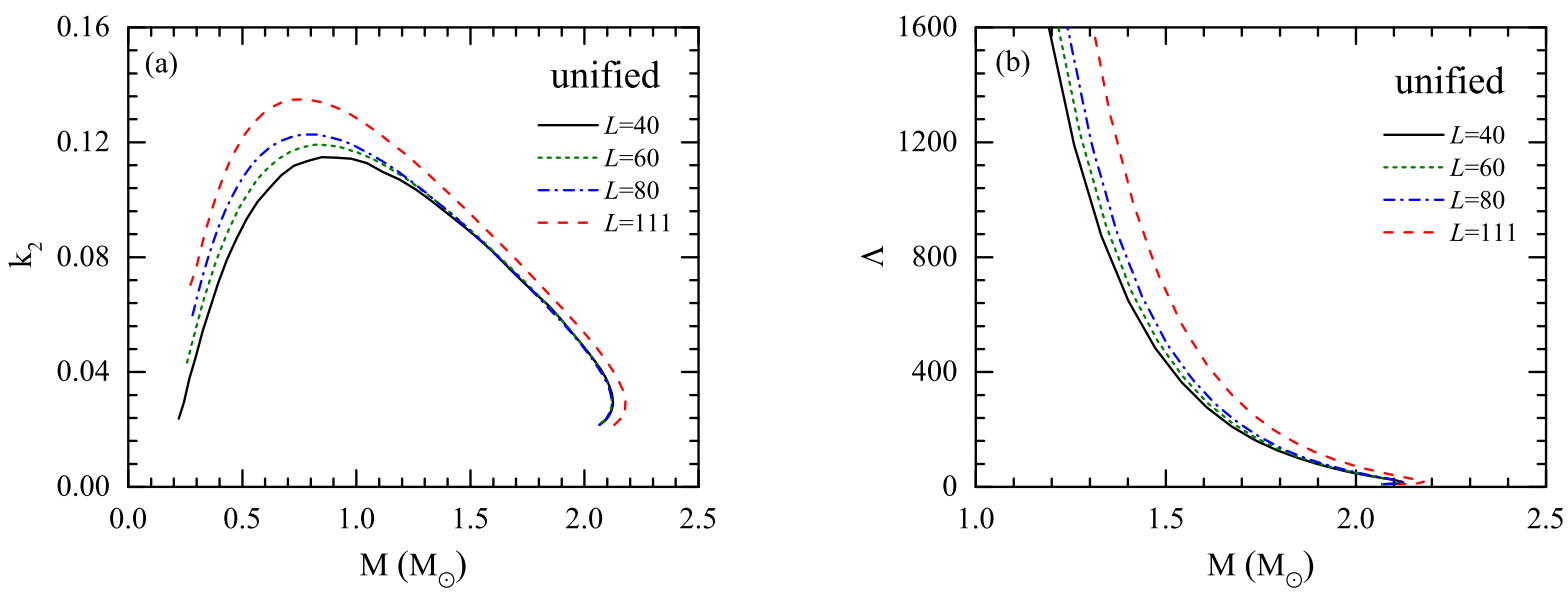

FIG. 5: (Color online) Love number $k_{2}$ and tidal deformability $\Lambda$ as a function of the neutron-star mass $M$ obtained using the unified EOSs shown in Fig. 2.

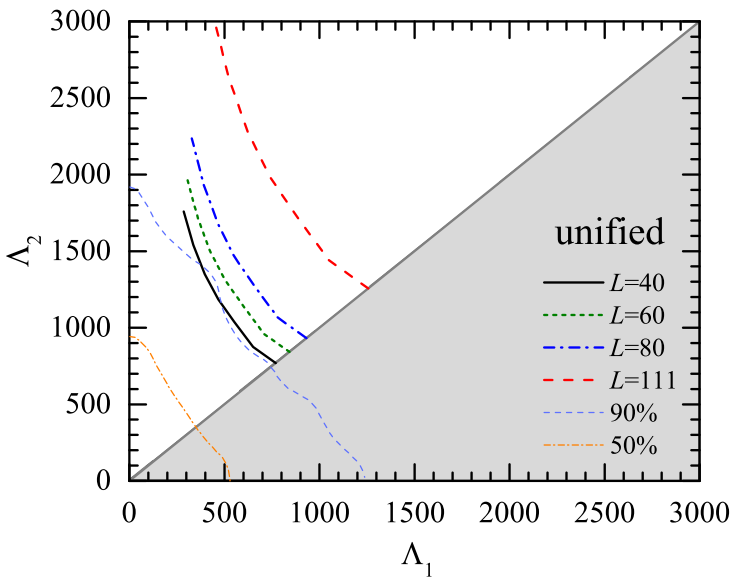

FIG. 6: (Color online) Tidal deformabilities $\Lambda_{1}$ vs $\Lambda_{2}$ of the two neutron stars in GW170817, using the unified EOSs with different slope parameters $L$. The $90 \%$ and $50 \%$ credible constraints from the latest analysis of GW170817 [2] are shown by thin dashed and dash-dotted lines, respectively.

ing observations are more compatible with an EOS having a smaller value of $L$. Therefore, the $\operatorname{TM} 1(L=40)$ model is more favored by the cooling observations than the $\operatorname{TM} 1(L=111)$ model.

We present, in Fig. 4, the resulting mass-radius relation with the set of unified EOSs. It is found that the maximum mass of neutron stars lies in the range of $2.12-$ $2.18 M_{\odot}$, which is compatible with the observational constraints of PSR J1614-2230 $\left(M=1.928 \pm 0.017 M_{\odot}\right)$ 3, 4], PSR J0348+0432 $\left(M=2.01 \pm 0.04 M_{\odot}\right)$ [5], and PSR J0740+6620 $\left(M=2.14_{-0.09}^{+0.10} M_{\odot}\right)[6]$. It is shown that the maximum mass is not very sensitive to the slope parameter $L$, but the radius obviously depends on the value of
$L$. We find that the radius of a canonical $1.4 M_{\odot}$ neutron $\operatorname{star}\left(R_{1.4}\right)$ is $\approx 14.21 \mathrm{~km}$ using the $\operatorname{TM} 1(L=111)$ model, while it reduces to $\approx 13.12 \mathrm{~km}$ with the $\operatorname{TM} 1(L=40)$ model. So far, the precise measurement of neutron-star radii is still a challenge for astrophysical observations, and no stringent constraints on the radius $R_{1.4}$ can be derived [44, 71]. The recent analysis of GW170817 data provides a constraint on the radius of a $1.4 M_{\odot}$ neutron star of $R_{1.4}<13.6 \mathrm{~km}[1]$. Many studies based on different approaches for the GW170817 event suggested a consistent upper limit for the radius of a $1.4 M_{\odot}$ neutron

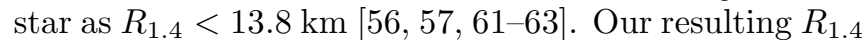
with a smaller $L$ is compatible with this constraint. It is noteworthy that the calculations of neutron-star radii are model dependent, as can be found in Refs. [9, 10, 32, 53]. In Ref. [53], quantum Monte Carlo calculations predict $R_{1.4}<12 \mathrm{~km}$ for $L \leq 45 \mathrm{MeV}$, which are much smaller than our results. Therefore, the slope parameter $L$ cannot be precisely constrained by observations of neutronstar radii due to the model dependency. On the other hand, the positive correlation between $L$ and $R_{1.4}$ is consistent among different models.

It is interesting to examine the correlation between the tidal deformability of neutron stars and the density dependence of nuclear symmetry energy. The tidal deformability is determined by the EOS through both the tidal Love number $k_{2}$ and the compactness parameter $C=M / R$, as shown in Eq. (11). We plot in Fig. 5 the tidal Love number $k_{2}$ (left panel) and the dimensionless tidal deformability $\Lambda$ (right panel) as a function of the neutron-star mass $M$. One can see that $k_{2}$ increases with the neutron-star mass and reaches its maximum value around $0.7-0.9 M_{\odot}$, and then decreases rapidly in the large-mass region. We find that there are significant differences in $k_{2}$ for a fixed $M$ between the EOSs with different slope parameters $L$, especially for smaller neutronstar masses. The model with a small $L$ predicts a small 
value of $k_{2}$, and therefore a small tidal deformability $\Lambda$ is achieved due to their relation in Eq. (11). It is shown that a clear $L$ dependence of the tidal deformability $\Lambda$ is observed, which comes from the $L$ dependence of both the tidal Love number $k_{2}$ and the radius $R$. The value of $\Lambda$ is very large for a small neutron-star mass due to its small compactness parameter. As the star mass increases, the tidal deformability $\Lambda$ decreases rapidly. For the canonical $1.4 M_{\odot}$ neutron star, we obtain $\Lambda=652$ using the $\operatorname{TM} 1(L=40)$ model, while it increases to $\Lambda=1047$ for the $\operatorname{TM} 1(L=111)$ model. The analysis of GW170817 data has placed a constraint on the tidal deformability of a $1.4 M_{\odot}$ neutron star, i.e., $\Lambda_{1.4}<800$ [1]. Hence, an EOS with a small symmetry energy slope like $L=40 \mathrm{MeV}$ is more favored than one with a large slope like $L=111$ $\mathrm{MeV}$.

In Fig. 6. we plot the tidal deformabilities $\Lambda_{1}$ vs $\Lambda_{2}$ of the two neutron stars in GW170817, using the unified EOSs with different slope parameters. $\Lambda_{1}$ and $\Lambda_{2}$ are the individual tidal deformabilities associated with the highmass $M_{1}$ and low-mass $M_{2}$ components of the binary, respectively. The curves are obtained by varying independently the high-mass component in the range $1.365 \leq$ $M_{1} / M_{\odot} \leq 1.60$, whereas the low-mass component is determined by keeping the chirp mass fixed at the observed value of $\mathcal{M}=\left(M_{1} M_{2}\right)^{3 / 5}\left(M_{1}+M_{2}\right)^{-1 / 5}=1.188 M_{\odot}[1]$. The $90 \%$ and $50 \%$ credible constraints from the latest analysis of GW170817 by LIGO and Virgo Collaborations [2] are shown by thin dashed and dash-dotted lines, respectively. Compared to the $90 \%$ confidence limit reported in the initial analysis of GW170817 [1], the present $90 \%$ credible constraint is considerably reduced. We can see that the curve obtained by the TM1 $(L=40)$ model is compatible with the $90 \%$ credible constraint, but other curves with larger $L$ are almost ruled out. The correlation between the tidal deformability and the slope parameter suggests that large values of $L$ are not favored by GW170817.

\section{B. Effects of the crust EOS}

We separately investigate the effects of crust and core EOSs on neutron-star properties. To examine the effect of the crust, we construct a set of nonunified EOSs by matching the same core EOS to different crust segments. The crust-core transition is determined by the crossing point of the two segments, where the crust and core have equal pressure and energy density. In Fig. 7 , we display the pressure $P$ as a function of the energy density $\varepsilon$ for the set of nonunified EOSs, where the TM1 $(L=40)$ model is used for the core and the inner crust is described by the models with different slope parameter $L$. It is shown that there are obvious differences in the inner crust region among these EOSs, whereas no difference exists both in the outer crust BPS EOS and in the core $\operatorname{TM} 1(L=40)$ EOS. The model with a large $L$ predicts a soft EOS of the inner crust, which is opposite to the behavior at high

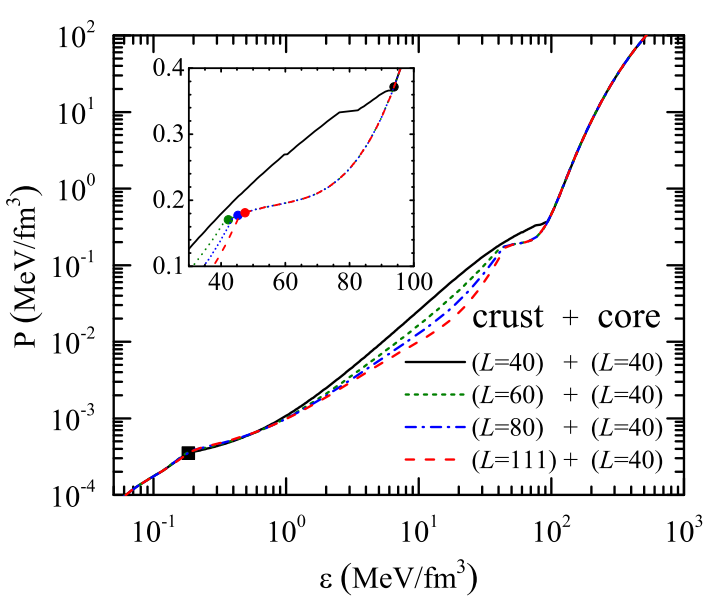

FIG. 7: (Color online) Pressure $P$ as a function of the energy density $\varepsilon$ obtained using the generated TM1 models with different $L$ for the inner crust and the $\operatorname{TM} 1(L=40)$ model for the core. The crust-core transition is indicated by the filled circles in the inset. The BPS EOS is adopted for the outer crust and the matching point is marked by the filled square.

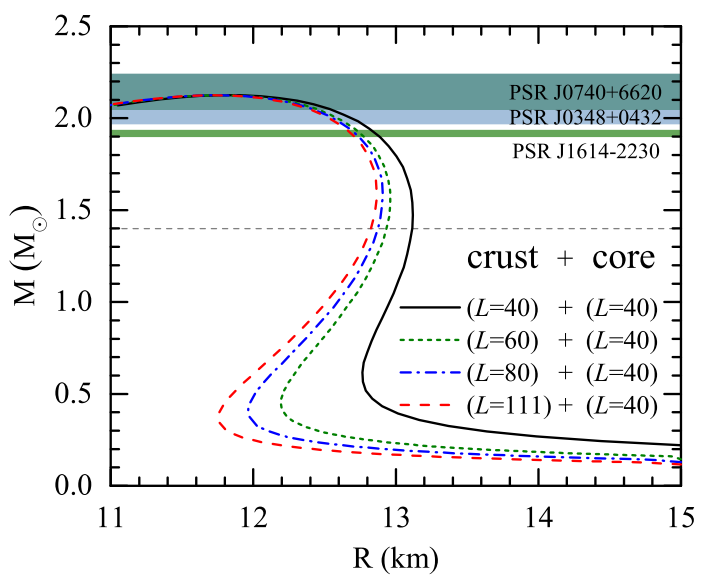

FIG. 8: (Color online) Mass-radius relations of neutron stars obtained using the nonunified EOSs shown in Fig. 7 The horizontal bars indicate the recent neutron-star mass measurements of PSR J1614-2230 [3, 4], PSR J0348+0432 [5], and PSR J0740+6620 [6].

densities (see Fig. 22). Therefore, the softest EOS considered here is the combination of the crust with $L=111$ $\mathrm{MeV}$ and the core with $L=40 \mathrm{MeV}$. The $L$ dependence of the EOS can be understood from the density dependence of the symmetry energy $E_{\text {sym }}$ shown in Fig. 1.

It is interesting to examine quantitatively the effect of the inner crust on neutron-star properties. In Fig. 8, we plot the mass-radius relation obtained using the set of nonunified EOSs. It is noticed that almost no difference 

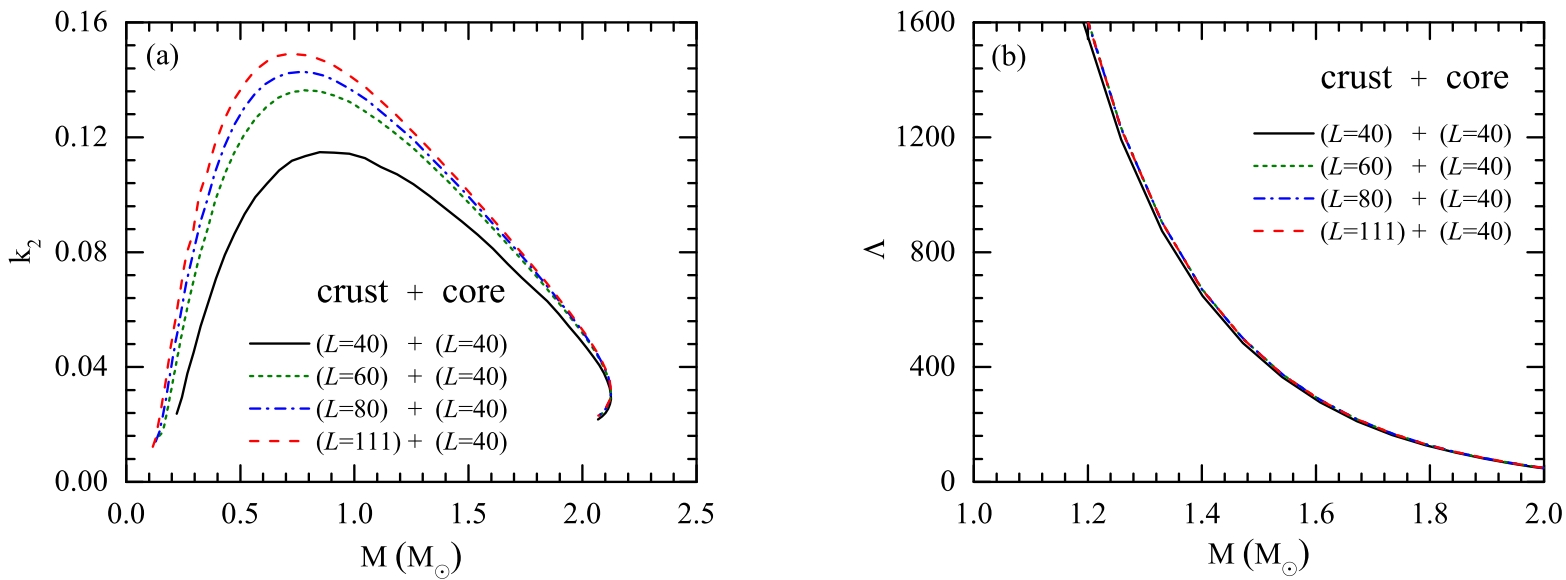

FIG. 9: (Color online) Love number $k_{2}$ and tidal deformability $\Lambda$ as a function of the neutron-star mass $M$ obtained using the nonunified EOSs shown in Fig. 7

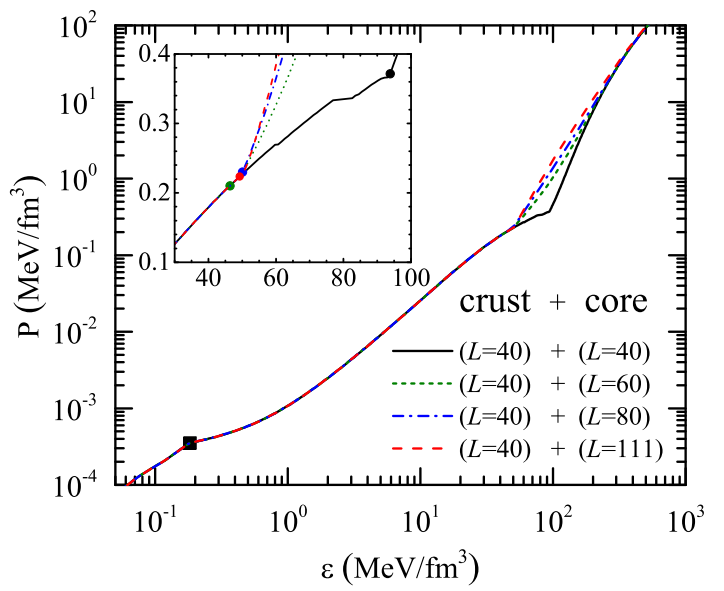

FIG. 10: (Color online) Pressure $P$ as a function of the energy density $\varepsilon$ obtained using the generated TM1 models with different $L$ for the core and $\operatorname{TM} 1(L=40)$ for the inner crust. The crust-core transition is indicated by the filled circles in the inset. The BPS EOS is adopted for the outer crust and the matching point is marked by the filled square.

is found for massive neutron stars when using different EOSs, which indicates the crust contribution is unimportant for a large mass star. On the other hand, the difference in the radius becomes more pronounced as the mass decreases. For the canonical $1.4 M_{\odot}$ neutron star, the radius $R_{1.4}$ changes from $\approx 13.12 \mathrm{~km}$ using the unified $\operatorname{TM} 1(L=40)$ EOS to $\approx 12.82 \mathrm{~km}$ when replacing the crust EOS with $\operatorname{TM} 1(L=111)$. This means that the difference in the crust EOS may lead to $\approx 0.3 \mathrm{~km}$ difference in $R_{1.4}$. Furthermore, it is found that a small $L$ of the crust corresponds to a large neutron-star radius, which is opposite to the $L$ dependence shown in Fig. (4) This is

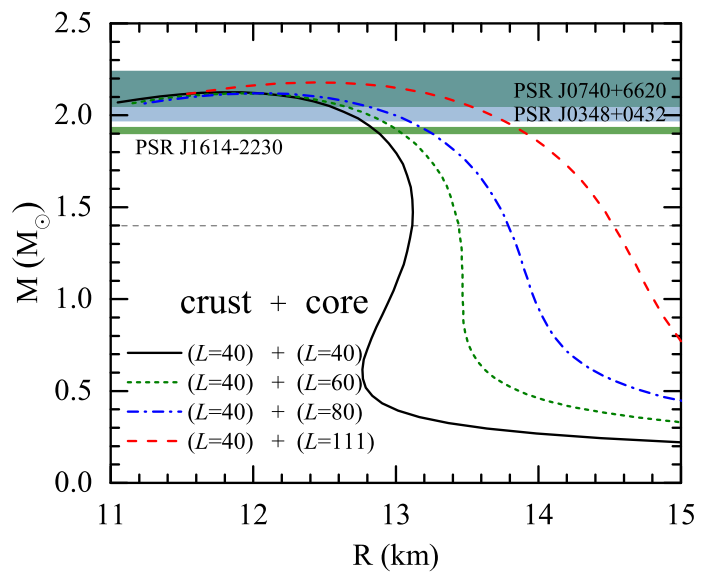

FIG. 11: (Color online) Mass-radius relations of neutron stars obtained using the nonunified EOSs shown in Fig. 10. The horizontal bars indicate the recent neutron-star mass measurements of PSR J1614-2230 [3, 4], PSR J0348+0432 [5], and PSR J0740+6620 [] ].

because the model with a small $L$ results in a hard EOS at subnuclear densities and a soft EOS at supernuclear densities. In the case of the unified EOS, the neutronstar radius is determined dominantly by the core EOS, where the crust EOS is less important. When the nonunified EOSs shown in Fig. 7 are employed, the differences in the radii come only from the inner crust segments. Therefore, the sensitivity of the radius to the crust EOS can be examined by using this set of nonunified EOSs.

To study the influence of the crust EOS on the tidal deformability of neutron stars, we show in Fig. 9 the tidal Love number $k_{2}$ (left panel) and the dimensionless tidal deformability $\Lambda$ (right panel) as a function of the 

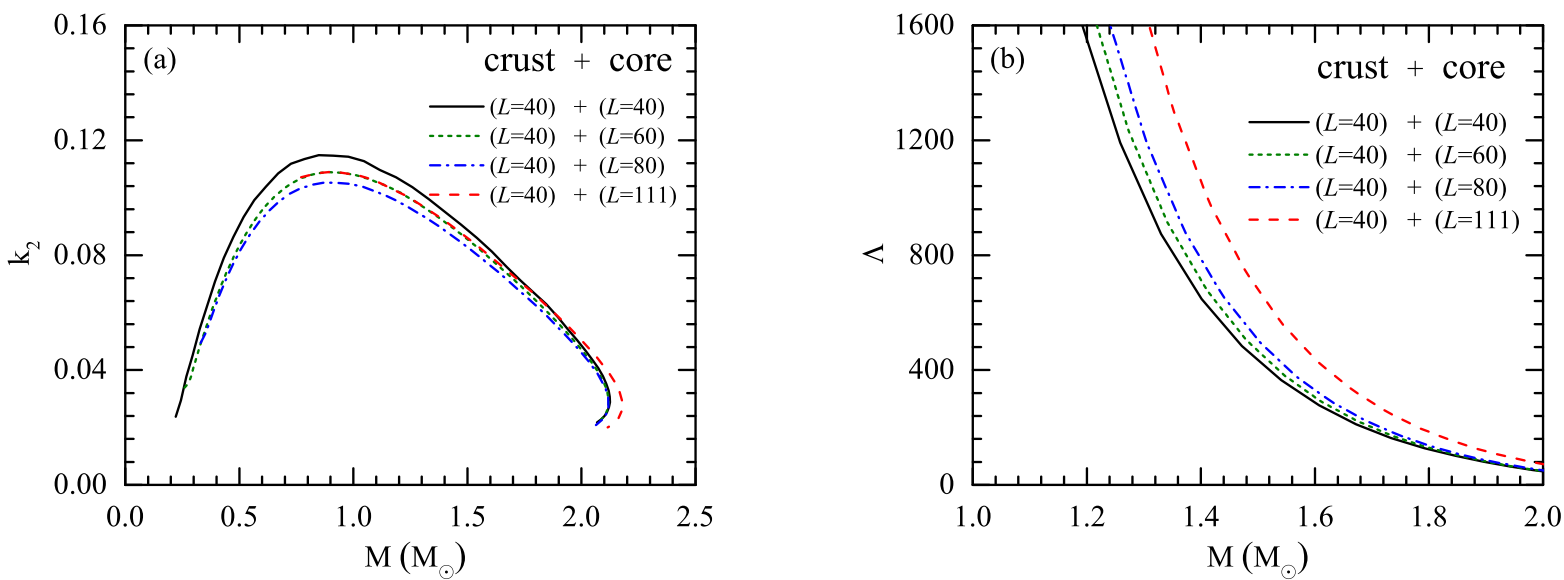

FIG. 12: (Color online) Love number $k_{2}$ and tidal deformability $\Lambda$ as a function of the neutron-star mass $M$ obtained using the nonunified EOSs shown in Fig. 10

neutron-star mass $M$, using the set of nonunified EOSs shown in Fig. 7. The behavior of $k_{2}$ in this case is very similar to that using unified EOSs, as shown in the left panel of Fig. 5. The maximum values of $k_{2}$ obtained using the non-unified EOSs are somewhat higher than corresponding results of unified EOSs. Although the same core EOS is adopted for all non-unified EOSs considered, significant differences are found in $k_{2}$ due to the difference of the inner crust. This implies that the tidal Love number $k_{2}$ is rather sensitive to the crust EOS. However, the tidal deformability $\Lambda$ shown in right panel of Fig. 9 is not so sensitive to the crust EOS. Comparing to $\Lambda$ obtained by the unified EOSs (see Fig. 5), the differences in $\Lambda$ when using the nonunified EOSs are much smaller. This is because $\Lambda$ depends on both the tidal Love number $k_{2}$ and the compactness parameter $C=M / R$. Due to opposite $L$ dependence of the radius $R$ shown in Figs. 4 and 8 , the enhancement of $\Lambda$ with $L$ contributed by $k_{2}$ is counteracted by the decrease of $R$ (equal to the increase of $C$ ) in the case of nonunified EOSs, but it is enhanced by the increase of $R$ for unified EOSs. Therefore, the $L$ dependence of $\Lambda$ shown in Fig. 5 is more pronounced than the one in Fig. 9 .

\section{Effects of the core EOS}

To examine the effect of the core EOS on neutronstar properties, we construct another set of nonunified EOSs by matching the same crust EOS to different core segments. Again, the crust-core transition is determined by the crossing point of the two segments. In Fig. 10, we display the pressure $P$ as a function of the energy density $\varepsilon$ for the set of nonunified EOSs using the BPS EOS for the outer crust, the $\operatorname{TM} 1(L=40)$ model for the inner crust, and the TM1 $(L=40,60,80,111)$ models for the core. It is shown that differences appear only in the core segments among these EOSs. The model with a large $L$ predicts a stiff EOS at high densities.

In Fig. 11] we plot the mass-radius relation obtained using the nonunified EOSs with different core segments. It is seen that the impact of the slope parameter $L$ of the core is rather obvious, especially on the radii of small mass neutron stars. For the canonical $1.4 M_{\odot}$ neutron star, the radius $R_{1.4}$ is $\approx 14.53 \mathrm{~km}$ in the case of nonunified EOS with the TM1 $(L=40)$ crust matching the $\operatorname{TM} 1(L=111)$ core, whereas it is reduced to $\approx 13.12 \mathrm{~km}$ when the TM1 $(L=40)$ core is adopted. The difference between these two cases is even larger than the one of unified EOSs shown in Fig. 4. This is because the combination of the $\operatorname{TM} 1(L=40)$ crust matching the TM1 $(L=111)$ core predicts the stiffest EOS among all combinations considered in this work. This can be understood from the density dependence of the symmetry energy, as shown in Fig. 1. We find that both the core and crust EOSs can significantly affect the neutron-star radii, as shown in Figs. 8 and [1], but their $L$ dependences are opposite.

To study the impact of the core EOS on the tidal deformability of neutron stars, we plot in Fig. 12 the tidal Love number $k_{2}$ (left panel) and the dimensionless tidal deformability $\Lambda$ (right panel) as a function of the neutronstar mass $M$, using the set of nonunified EOSs shown in Fig. 10. It is found that $k_{2}$ is insensitive to the slope parameter $L$ of the core, which is different from the behavior shown in Figs. 5 and 9. This indicates that the tidal Love number $k_{2}$ is mainly determined by the crust EOS. On the other hand, the tidal deformability $\Lambda$ shown in right panel of Fig. 12 is clearly dependent on the slope parameter $L$ of the core. The behavior of $\Lambda$ in this case is very similar to that using unified EOSs, as shown in the right panel of Fig. 5. With increasing $L$ of the core, the enhancement of $\Lambda$ shown in Fig. 12 is mostly contributed from the decrease of the compactness parameter $C$, because $k_{2}$ is insensitive to the slope parameter $L$ of the 
core. This is different from the case of unified EOSs, where the $L$ dependence of $\Lambda$ shown in Fig. [5] is determined by both $C$ and $k_{2}$. To analyze the effects of the crust and core EOSs in more detail, we present in Table III some basic properties of neutron stars obtained using different combinations of the crust and core segments. It is found that $M_{\max }$ are determined by the core EOS, whereas the properties of a canonical $1.4 M_{\odot}$ neutron star are affected by both the crust and core EOSs. It is noticeable that the crust with different $L$ may result in $\approx 0.3 \mathrm{~km}$ difference in the radius $R_{1.4}$ and $\approx 0.2-0.4 \mathrm{~km}$ difference in the crust thickness $\Delta R_{1.4}^{\text {crust }}$. Although $k_{2}^{1.4}$ and $C_{1.4}$ are affected by the crust EOS, the calculated $\Lambda_{1.4}$ is not so sensitive to the crust EOS.

\section{CONCLUSIONS}

In this work, we constructed a set of unified EOSs based on RMF models with different slope parameters $L$. We performed the self-consistent Thomas-Fermi calculations for pasta phases appearing in the inner crust and then determined the crust-core transition by comparing the energy densities between pasta phases and homogeneous matter. It was found that the model with a small $L$ predicts a large crust-core transition density. By applying the set of unified EOSs in neutron-star calculations, some correlations between the symmetry energy slope $L$ and neutron-star properties were observed. It was found that a small $L$ corresponds to a small neutron-star radius and therefore a small tidal deformability, which is favored by the recent analysis of the GW170817 event.

To separately investigate the effects of crust and core EOSs on neutron-star properties, we constructed two sets of nonunified EOSs: (1) the same core EOS matching different crust EOSs; (2) the same crust EOS matching different core EOSs. It was observed that different crust EOSs could lead to significant difference in neutronstar radii. For the canonical $1.4 M_{\odot}$ neutron star, the radius $R_{1.4}$ changes from $\approx 13.12 \mathrm{~km}$ with the unified $\operatorname{TM} 1(L=40)$ EOS to $\approx 12.82 \mathrm{~km}$ when replacing the crust EOS with $\operatorname{TM} 1(L=111)$. Therefore, the uncertainty in $R_{1.4}$ induced by different crust EOSs considered here is $\approx 0.3 \mathrm{~km}$. On the other hand, the influence of the core EOS on neutron-star radii is more pronounced than the one of the crust EOS. The uncertainty in $R_{1.4}$ induced by different core EOSs is $\approx 1.4 \mathrm{~km}$. We noticed that the $L$ dependence of neutron-star radii obtained using the two sets of nonunified EOSs is opposite, which could be understood from the density dependence of the symmetry energy.

We studied the tidal deformability of neutron stars using the two sets of nonunified EOSs, in order to examine the effects of crust and core EOSs separately. It was found that the effect of the core EOS on the tidal deformability $\Lambda$ is more significant than the one of the crust EOS. In fact, the tidal Love number $k_{2}$ is mainly determined by the crust EOS and is insensitive to the slope parameter $L$ of the core. With increasing $L$ of the core, the enhancement of $\Lambda$ is mostly contributed from the increase of the neutron-star radius $R$. On the other hand, the crust EOS could significantly affect both the Love number $k_{2}$ and the radius $R$. However, the enhancement of $k_{2}$ with $L$ is largely counteracted by the decrease of $R$. Therefore, the resulting tidal deformability $\Lambda$ is not so sensitive to the crust EOS. We concluded that both the crust and core EOSs could significantly affect neutron-star properties such as the radius and tidal deformability. It is likely that the nuclear model with a small symmetry energy slope is favored by various observational constraints.

\section{Acknowledgment}

We would like to acknowledge helpful discussions with K. Sumiyoshi, K. Nakazato, K. Oyamatsu, and H. Toki. This work was supported in part by the National Natural Science Foundation of China (Grants No. 11675083, No. 11775119, and No. 11805115). 
TABLE III: Properties of neutron stars for different combinations of the crust and core EOSs. $M_{\max }$ is the maximum mass of neutron stars. $R_{1.4}$ and $\Delta R_{1.4}^{\text {crust }}$ denote respectively the radius and crust thickness of a canonical $1.4 M_{\odot}$ neutron star. $k_{2}^{1.4}, C_{1.4}$, and $\Lambda_{1.4}$ are the Love number, the compactness parameter, and the tidal deformability for a $1.4 M_{\odot}$ neutron star, respectively.

\begin{tabular}{lccccccc}
\hline \hline EOS & $\begin{array}{c}\text { Combination } \\
\text { TM1 }\end{array}$ & $\begin{array}{c}M_{\max } \\
\text { crust+core }\end{array}$ & $\begin{array}{c}R_{1.4} \\
\left(M_{\odot}\right)\end{array}$ & $\begin{array}{c}\Delta R_{1.4}^{\text {crust }} \\
(\mathrm{km})\end{array}$ & $k_{2}^{1.4}$ & $C_{1.4}$ & $\Lambda_{1.4}$ \\
\hline unified & $(L=40)+(L=40)$ & 2.12 & 13.12 & 1.25 & 0.095 & 0.158 & 652 \\
unified & $(L=111)+(L=111)$ & 2.18 & 14.21 & 1.27 & 0.103 & 0.145 & 1047 \\
\hline nonunified & $(L=40)+(L=111)$ & 2.18 & 14.53 & 1.44 & 0.092 & 0.142 & 1050 \\
nonunified & $(L=111)+(L=40)$ & 2.12 & 12.82 & 0.84 & 0.110 & 0.161 & 671 \\
\hline \hline
\end{tabular}

[1] B. P. Abbott et al. (LIGO Scientifc Collaboration and Virgo Collaboration), Phys. Rev. Lett. 119, 161101 (2017).

[2] B. P. Abbott et al. (LIGO Scientifc Collaboration and Virgo Collaboration), Phys. Rev. Lett. 121, 161101 (2018).

[3] P. B. Demorest, T. Pennucci, S. M. Ranson, M. S. E. Roberts, and J. W. T. Hessels, Nature (London) 467, 1081 (2010).

[4] E. Fonseca et al., Astrophys. J. 832, 167 (2016).

[5] J. Antoniadis et al., Science 340, 6131 (2013).

[6] H. T. Cromartie et al., arXiv:1904.06759.

[7] J. M. Lattimer and M. Prakash, Science 304, 536 (2004).

[8] N. Chamel and P. Haensel, Living Rev. Relativ. 11, 10 (2008).

[9] J. M. Lattimer and M. Prakash, Phys. Rep. 621, 127 (2016).

[10] M. Oertel, M. Hempel, T. Klähn, and S. Typel, Rev. Mod. Phys. 89, 015007 (2017).

[11] J. M. Pearson, S. Goriely, and N. Chamel, Phys. Rev. C 83, 065810 (2011).

[12] D. G. Ravenhall, C. J. Pethick, and J. R. Wilson, Phys. Rev. Lett. 50, 2066 (1983).

[13] S. S. Avancini, D. P. Menezes, M. D. Alloy, J. R. Marinelli, M. M. W. Moraes, and C. Providência, Phys. Rev. C 78, 015802 (2008).

[14] S. S. Bao and H. Shen, Phys. Rev. C 89, 045807 (2014).

[15] F. Grill, C. Providência, and S. S. Avancini, Phys. Rev. C 85, 055808 (2012).

[16] M. Okamoto, T. Maruyama, K. Yabana, and T. Tatsumi, Phys. Rev. C 88, 025801 (2013).

[17] S. S. Bao and H. Shen, Phys. Rev. C 91, 015807 (2015).

[18] F. J. Fattoyev, C. J. Horowitz, and B. Schuetrumpf, Phys. Rev. C 95, 055804 (2017).

[19] N. K. Glendenning, Phys. Rev. D 46, 1274 (1992).

[20] N. K. Glendenning, Phys. Rep. 342, 393 (2001).

[21] F. Weber, Prog. Part. Nucl. Phys. 54, 193 (2005).

[22] K. Masuda, T. Hatsuda, and T. Takatsuka, Astrophys. J. 764, 12 (2013); Prog. Theor. Exp. Phys. 2013, 073D01 (2013).

[23] A. Bhattacharyya, I. N. Mishustin, and W. Greiner, J. Phys. G 37, 025201 (2010).

[24] N. Yasutake, R. Łastowiecki, S. Benić, D. Blaschke, T. Maruyama, and T. Tatsumi, Phys. Rev. C 89, 065803
(2014).

[25] W. M. Spinella, F. Weber, G. A. Contrera, and M. G. Orsaria, Eur. Phys. J. A 52, 61 (2016).

[26] X. H. Wu and H. Shen, Phys. Rev. C 99, 065802 (2019).

[27] F. Douchin and P. Haensel, Astron. Astrophys. 380, 151 (2001).

[28] H. Shen, Phys. Rev. C 65, 035802 (2002).

[29] T. Miyatsu, S. Yamamuro, and K. Nakazato, Astrophys. J. 777, 4 (2013).

[30] A. F. Fantina, N. Chamel, J. M. Pearson, and S. Goriely, Astron. Astrophys. 559, A128 (2013).

[31] B. K. Sharma, M. Centelles, X. Viñas, M. Baldo, and G. F. Burgio, Astron. Astrophys. 584, A103 (2015).

[32] M. Fortin, C. Providência, Ad. R. Raduta, F. Gulminelli, J. L. Zdunik, P. Haensel, and M. Bejger, Phys. Rev. C 94, 035804 (2016).

[33] B. D. Serot and J. D. Walecka, Adv. Nucl. Phys. 16, 1 (1986).

[34] J. Meng, H. Toki, S. G. Zhou, S. Q. Zhang, W. H. Long, and L. S. Geng, Prog. Part. Nucl. Phys. 57, 470 (2006).

[35] Y. Sugahara and H. Toki, Nucl. Phys. A 579, 557 (1994).

[36] B. A. Li, L. W. Chen, and C. M. Ko, Phys. Rep. 464, 113 (2008).

[37] C. J. Horowitz and J. Piekarewicz, Phys. Rev. Lett. 86, 5647 (2001).

[38] C. Ducoin, J. Margueron, and C. Providência, Europhys. Lett. 91, 32001 (2010).

[39] K. Oyamatsu and K. Iida, Phys. Rev. C 75, 015801 (2007).

[40] R. Cavagnoli, D. P. Menezes, and C. Providência, Phys. Rev. C 84, 065810 (2011).

[41] C. Providência and A. Rabhi, Phys. Rev. C 87, 055801 (2013).

[42] I. Tews, J. M. Lattimer, A. Ohnishi, and E. E. Kolomeitsev, Astrophys. J. 848, 105 (2017).

[43] K. Hebeler, J. M. Lattimer, C. J. Pethick, and A. Schwenk, Astrophys. J. 773, 11 (2013).

[44] J. M. Lattimer and A. W. Steiner, Eur. Phys. J. A 50, 40 (2014).

[45] G. Hagen, A. Ekström, C. Forssén, G. R. Jansen, W. Nazarewicz, T. Papenbrock, K. A. Wendt, S. Bacca, N. Barnea, B. Carlsson, C. Drischler, K. Hebeler, M. HjorthJensen, M. Miorelli, G. Orlandini, A. Schwenk, and J. Simonis, Nat. Phys. 12, 186 (2015) 
[46] P. Danielewicz and J. Lee, Nucl. Phys. A 922, 1 (2014).

[47] X. Roca-Maza, X. Viñas, M. Centelles, B. K. Agrawal, G. Colò, N. Paar, J. Piekarewicz, and D. Vretenar, Phys. Rev. C 92, 064304 (2015).

[48] J. Birkhan, M. Miorelli, S. Bacca, S. Bassauer, C. A. Bertulani, G. Hagen, H. Matsubara, P. von NeumannCosel, T. Papenbrock, N. Pietralla, V. Yu. Ponomarev, A. Richter, A. Schwenk, and A. Tamii, Phys. Rev. Lett. 118, 252501 (2017).

[49] P. Danielewicz, P. Singh, and J. Lee, Nucl. Phys. A 958, 147 (2017).

[50] S. S. Bao, J. N. Hu, Z. W. Zhang, and H. Shen, Phys. Rev. C 90, 045802 (2014).

[51] H. Shen, H. Toki, K. Oyamatsu, and K. Sumiyoshi, Astrophys. J. Suppl. 197, 20 (2011).

[52] F. J. Fattoyev, C. J. Horowitz, J. Piekarewicz, and G. Shen, Phys. Rev. C 82, 055803 (2010).

[53] S. Gandolfi, J. Carlson, and S. Reddy, Phys. Rev. C 85, 032801 (2012).

[54] G. Baym, C. Pethick, and P. Sutherland, Astrophys. J. 170, 299 (1971).

[55] N. B. Zhang, B. A. Li, J. Xu, Astrophys. J. 859, 90 (2018).

[56] I. Tews, J. Margueron, and S. Reddy, Phys. Rev. C 98, 045804 (2018).

[57] Z. Y. Zhu, E. P. Zhou, and A. Li, Astrophys. J. 862, 98 (2018).

[58] E. Annala, T. Gorda, A. Kurkela, and A. Vuorinen, Phys. Rev. Lett. 120, 172703 (2018).
[59] Y. Lim and J. W. Holt, Phys. Rev. Lett. 121, 062701 (2018).

[60] D. Radice, A. Perego, F. Zappa, and S. Bernuzzi, Astrophys. J. Lett. 852, L29 (2018).

[61] S. De, D. Finstad, J. M. Lattimer, D. A. Brown, E. Berger, and C. M. Biwer, Phys. Rev. Lett. 121, 091102 (2018).

[62] F. J. Fattoyev, J. Piekarewicz, and C. J. Horowitz, Phys. Rev. Lett. 120, 172702 (2018).

[63] T. Malik, N. Alam, M. Fortin, C. Providência, B. K. Agrawal, T. K. Jha, B. Kumar, and S. K. Patra, Phys. Rev. C 98, 035804 (2018).

[64] V. Dexheimer, R. O. Gomes, S. Schramm, and H. Pais, J. Phys. G 46, 034002 (2019).

[65] P. G. Krastev and B. A. Li, J. Phys. G 46, 074001 (2019).

[66] T. Hinderer, Astrophys. J. 677, 1216 (2008); 697, 964(E) (2009).

[67] S. Postnikov, M. Prakash, and J. M. Lattimer, Phys. Rev. D 82, 024016 (2010).

[68] H. Pais, W. G. Newton, and J. R. Stone, Phys. Rev. C 90, 065802 (2014).

[69] J. M. Lattimer, C. J. Pethick, M. Prakash, P. Haensel, Phys. Rev. Lett. 66, 2701 (1991).

[70] R. Negreiros, L. Tolos, M. Centelles, A. Ramos, and V. Dexheimer, Astrophys. J. 863, 104 (2018).

[71] P. Haensel, M. Bejger, M. Fortin, and L. Zdunik, Eur. Phys. J. A 52, 59 (2016). 